Pacific Journal of Mathematics

FIXED ELEMENTS OF JORDAN AUTOMORPHISMS O 


\title{
FIXED ELEMENTS OF JORDAN AUTOMORPHISMS OF ASSOCIATIVE RINGS
}

\author{
W. S. Martindale, III and Susan Montgomery
}

Let $R$ be an associative ring, and let $G$ be a group of Jordan automorphisms of $R$. Let $R^{a}$ be the set of elements in $R$ fixed by all $g \in G$; that is, $R^{G}=\left\{x \in R \mid x^{g}=x\right.$, all $\left.g \in G\right\}$. Although $R^{a}$ is not necessarily a subring of $R$, it is a Jordan subring of $R$. In this paper, we will study the relationship between the structure of $R^{G}$ as a Jordan ring and the structure of $R$, where $G$ will usually be a finite group of order $|G|$ and the ring $R$ has no additive $|G|$-torsion.

More specifically, under the above hypothesis, we show that the prime radical of $R^{G}$ is the contraction of the prime radical of $R$, that if $R^{G}$ satisfies a polynomial identity then so does $R$, and if $R^{G}$ is nil of bounded index then so is $R$. With the additional assumption that $|G| R=R$, we show that the Jacobson radical of $R^{G}$ is the contraction of the Jacobson radical of $R$. We also obtain various relationships between ideals of $R^{G}$ and ideals of $R$.

Many of these results were already known in two major special cases of Jordan automorphisms: the case of ordinary (associative) automorphisms of $R$, and the case when $R$ has an involution. Moreover, our hypothesis that $R$ has no additive $|G|$-torsion is necessary because of existing counterexamples in these two cases. The known results and examples will be discussed in the relevant section, as each topic arises.

We now establish our terminology. By an automorphism of $R$ we will mean an ordinary automorphism of $R$ as an associative ring; we let $\operatorname{Aut}(R)$ denote the group of automorphisms $R$. If $A$ is an additive subgroup of $R, A$ is a (quadratic) Jordan subring of $R$ if $A$ is closed under squares (that is, $x^{2} \in A$ if $x \in A$ ) and under the quadratic operator $x U_{y}=y x y$ (as is well known, if $2 R=R$ this definition is equivalent to $A$ being closed under the single linear operation $a \cdot b=1 / 2(a b+b a)$. When we wish to consider $R$ itself as a Jordan ring, we will denote it by $R^{+}$. A mapping $\phi: R \rightarrow R^{\prime}$ of the rings $R$ and $R^{\prime}$ is a Jordan homomorphism if $\phi$ preserves the structure of $A$ as a Jordan ring; that is, $\phi$ is additive, $\phi\left(x^{2}\right)=\phi(x)^{2}$, all $x \in R$, and $\phi(y x y)=\phi(y) \phi(x) \phi(y)$, all $x, y \in R$. A Jordan automorphism of $R$ is simply a Jordan homomorphism which is also one-to-one and onto; we let $\operatorname{Aut}_{J}(R)$ denote the group of all Jordan automorphisms of $R$. If $G$ is a subgroup of $\operatorname{Aut}_{J}(R)$, then clearly $R^{G}$ is a Jordan subring of $R$. 
Now say that $G$ is finite with $|G|=n$. For $x \in R$, the trace of $x$ is $\operatorname{tr}_{G}(x)=\sum_{g \in G} x^{g}$. If there is no ambiguity about which group is involved, we simply write $\operatorname{tr}_{G}(x)=\operatorname{tr}(x)$. Note that $\operatorname{tr}(x) \in R^{G}$. When $R$ has an involution *, and $G=\{e, *\}$, we say that $G$ is generated by the involution. In this situation, $R^{G}=\left\{x \in R \mid x^{*}=x\right\}=S_{R}$, the symmetric elements in $R$.

If $I$ is an ideal of $R$, we say that $I$ is G-invariant if $I^{g} \subseteq I$, for all $g \in G$. When $I$ is $G$-invariant, $\bar{R}=R / I$ has an induced group of automorphisms, given as follows: for $g \in G$, define $\bar{g}$ by $(x+I)^{\bar{g}}=$ $x^{g}+I$. Let $K$ be the kernel of the mapping $g \rightarrow \bar{g}$, and let $\bar{G}=G / K$. Then $\bar{G}$ is a group of automorphisms of $\bar{R}$. Clearly $\overline{R^{G}} \subseteq \bar{R}^{\bar{a}}$, where $\overline{R^{G}}$ denotes the image of $R^{G}$ in $\bar{R}$.

We remark that more can be said when $G$ is finite: namely, if $|G|=n$, then $n \bar{R}^{\bar{G}} \subseteq \overline{\operatorname{tr}(R)} \cong \bar{R}^{\bar{G}}$. For, choose $\bar{x} \in \bar{R}^{\bar{\sigma}}$. Then $n \bar{x}=$ $|K||\bar{G}| \bar{x}=|K| \sum_{g \in G} \bar{x}^{\bar{g}}=\sum_{g \in G}|K| \bar{x}^{\bar{g}}=\overline{\sum_{g \in G}} x^{g}$, since each coset of $K$ in $G$ has precisely $|K|$ elements. Thus $n \bar{x}=\overline{\operatorname{tr}(x)} \in \overline{R^{G}}$. Finally, if $n$ is a bijection on $\bar{R}$ (that is, $n \bar{R}=\bar{R}$ and $\bar{R}$ has no additive $n$-torsion), equality will hold: $\bar{R}^{\bar{G}}=\overline{R^{G}}$.

1. Herstein's theorem and its consequences. Of fundamental importance in what follows is the following theorem of I. N. Herstein $[4$, p. 50].

THEOREM 1.1. Let $\phi: R \rightarrow R^{\prime}$ be a Jordan homomorphism of $R$ onto a prime ring $R^{\prime}$. Then $\phi$ is either a homomorphism or an anti-homomorphism.

Even when $R$ is not prime, Herstein's theorem has the following consequence for prime ideals:

COROLLARY 1.2. Let $\phi$ be a Jordan automorphism of $R$ and let $P$ be a prime ideal of $R$. Then $P^{\phi}$ is a prime ideal of $R$. Moreover, the prime rings $R / P$ and $R / P^{\phi}$ are either isomorphic or antiisomorphic.

Proof. Let $f: R \rightarrow R / P$ be the usual quotient map. Then, since $\phi^{-1}$ is also a Jordan automorphism of $R$, the composition $f \circ \phi^{-1}: R \rightarrow R / P$ is a Jordan homomorphism onto a prime ring, so by Herstein's theorem is either a homomorphism or an anti-homomorphism. Now $P^{\phi}=\left(f \circ \phi^{-1}\right)^{-1}(\overline{0})$, the inverse image of $(\overline{0})$ under a homomorphism or anti-homomorphism, and so $P^{\phi}$ is a prime ideal.

Now, $R / P^{\phi}$ is a prime ring by the above, and the mapping $\psi: R / P \rightarrow R / P^{\phi}$ given by $\psi(x+P)=x^{\phi}+P^{\phi}$ is a Jordan isomorphism 
of $R / P$ and $R / P^{\phi}$. Thus, $\psi$ is an isomorphism or anti-isomorphism by Herstein's theorem.

The next corollary will enable us, when $R$ is prime, to reduce to the cases of automorphisms and involutions.

CoROLLARY 1.3. Let $R$ be a prime ring, and $G$ a group of Jordan automorphisms of $R$. Let $H$ be the subgroup of $G$ consisting of all automorphisms. If $H \neq G$, then $[G: H]=2$. Moreover, $G / H$ induces an involution * on the associative ring $R^{H}$, as follows: choose $g \in G$, $g \notin H$, and let $x^{*}=x^{g}$, for any $x \in R^{H}$. The involution is independent of the choice of $g$, and the set of symmetric elements $S_{R^{H}}$ of $R^{H}$ under * is precisely the set $R^{G}$.

Proof. By Herstein's theorem, every element of $G$ is an automorphism or anti-automorphism, so if $H \neq G$, clearly $[G: H]=2$. The rest is straightforward.

Thus, when $R$ is prime, the class of Jordan rings arising as fixed elements of Jordan automorphisms is simply the class of symmetric elements in rings with involution. It is not known whether this is true in general, and we state it formally:

Question 1.4. For any ring $R$, and $G$ a group of Jordan automorphisms of $R$ does there exist a ring $R^{\prime}$ with involution such that $R^{G} \cong S_{R^{\prime}}$ ?

A question closely related to Question 1.4 is the following: to what extent can Herstein's theorem be generalized to semi-prime rings? One might hope that if $\phi$ were a Jordan automorphism of a semiprime ring $R$, then $\phi$ could be written as a sum $\phi=\phi_{1}+\phi_{2}$, where $\phi_{1}$ is a homomorphism and $\phi_{2}$ is an anti-homomorphism of $R$ to itself, and such that $\phi_{1}(R) \cap \phi_{2}(R)=(0)$. Assume for the moment that this were true, and let $A_{1}=\phi^{-1}\left(\phi_{1}(R)\right)$ and $A_{2}=\phi^{-1}\left(\phi_{2}(R)\right)$. Then one can check that $R=A_{1} \oplus A_{2}$, that $A_{i}$ is $\phi$-invariant, and that $\phi$ restricted to $A_{i}$ is just $\phi_{i}$. That is, the ring $R$ could be decomposed as a direct sum in such a way that $\phi$ acts as an automorphism on one component and as an anti-automorphism on the other.

That this is false can be seen from the following example: Let $T$ be any simple, noncommutative ring with an involution *, and let $R_{n}=\sum_{i=1}^{n} \oplus T_{i}$, where $T_{i}=T$, for each positive integer $n$. Define $\dot{\phi}_{n}: R_{n} \rightarrow R_{n}$ by $\phi_{n}\left(a_{1}, \cdots, a_{n}\right)=\left(a_{n}^{*}, a_{1}, \cdots, a_{n-1}\right)$. Then $\phi_{n}$ is a Jordan automorphism of $R_{n}$ which is neither a homomorphism nor an antihomomorphism, and $R_{n}$ cannot be written as a direct sum as desired since it has no nontrivial $\phi_{n}$-invariant ideals. Moreover, if we let 
$R=\prod_{n=1}^{\infty} R_{n}$, and let $\phi$ be given componentwise by $\phi_{n}$ on $R_{n}$, then $R$ is semi-prime but no power of $\phi$ is an automorphism. Thus, if it exists, an appropriate extension of Herstein's theorem would have to take some other form.

2. The prime radical. The main result of this section is that the prime radical of $R^{G}$ is the contraction to $R^{G}$ of the prime radical of $R$. If $A$ is a Jordan ring, an ideal $P$ of $A$ is prime if whenever $V U_{W} \subseteq P, V, W$ ideals of $A$, then either $V \subseteq P$ or $W \subseteq P$. The prime radical of $A$, which we shall denote by $P(A)$, is defined as the intersection of the prime ideals of $A$, and $A$ is semi-prime if $P(A)=(0)$ [11]. We shall also denote the prime radical of $R$ as an associative ring by $P(R)$; there is no ambiguity in this notation, for $P(R)=$ $P\left(R^{+}\right)$by a theorem of Erickson and Montgomery [3].

When $G$ is generated by an involution, and $R^{G}=S_{R}$, it was also proved by Erickson and Montgomery that $P\left(S_{R}\right)=P(R) \cap S_{R}$ [3], which is a special case of what we prove here. We will assume throughout this section that the group $G$ of Jordan automorphisms is finite, with $|G|=n$, and that $R$ has no additive $n$-torsion. Without this hypothesis, our desired result that $P\left(R^{G}\right)=P(R) \cap R^{G}$ is false (see the example in [10]).

Lemma 2.1. Let $R$ be n-torsion free. Then

(1) $R / P(R)$ is n-torsion free.

(2) $P(R)=\bigcap_{\alpha} P_{\alpha}$, where the $P_{\alpha}$ are all prime ideals of $R$ such that $R / P_{\alpha}$ is n-torsion free.

Proof. (1) We use the characterization of $P(R)$ as the set of elements $b \in R$ such that every $m$-sequence beginning with $b$ contains 0 [5, p. 196]. To show that $R / P(R)$ is $n$-torsion free, it will suffice to show that if $n b \in P(R)$, then $b \in P(R)$. Choose any $m$-sequence $\left\{a_{i}\right\}$ beginning with $b=a_{0}$. Then $\left\{n^{2^{i}} a_{i}\right\}$ is an $m$-sequence beginning with $n b \in P(R)$, so must contain 0 . Since $R$ is $n$-torsion free, $n^{2^{k}} a_{k}=0$ implies $a_{k}=0$, and thus $b \in P(R)$.

(2) Clearly $P(R)=\left(\bigcap_{\alpha} P_{\alpha}\right) \cap\left(\bigcap_{\beta} P_{\beta}\right)$ where $\left\{P_{\alpha}\right\}$ are prime ideals with $R / P_{\alpha} n$-torsion free and $\left\{P_{\beta}\right\}$ are prime ideals with $n R \subseteq P_{\beta}$. Thus if $x \in \bigcap_{\alpha} P_{\alpha}$, then $n x \in \bigcap_{\alpha} P_{B}$ also, and so $n x \in P(R)$. By (1), $x \in P(R)$ and so $\bigcap_{\alpha} P_{\alpha}=P(R)$.

Lemma 2.2. Let $A$ be a Jordan subring of $R$. Then $P(R) \cap$ $A \subseteq P(A)$.

Proof. The proof proceeds exactly as in [3, Lemma 1]. We provide it for completeness. 
Let $\mathscr{C}=$ ideals $M$ of $R \mid M \cap A \subseteq P(A)\}$. By Zorn's lemma, we may choose a maximal element $N \in \mathscr{M}$. We claim that $N$ is a semiprime ideal. For, say that $(J / N)^{2}=(\overline{0})$ in $R / N$; then $J^{2} \cong N$. Thus $(J \cap A) U_{(J \cap A)} \subseteq A U_{(J \cap A)} \cong A \cap R U_{J} \subseteq A \cap J^{2} \cong A \cap N \subseteq P(A)$. This implies that $J \cap A \subseteq P(A)$, since $P(A)$ is a semi-prime ideal, and hence $J \in \mathscr{M}$. By the maximality of $N, J=N$, and thus $N$ is a semi-prime ideal. It follows that $P(R) \subseteq N$, and so $P(R) \cap A \subseteq N \cap A \subseteq P(A)$.

We are now able to prove our desired result when $G$ consists of automorphisms. The proof uses a theorem of Bergman and Isaacs [2], which asserts that if $R$ is $|G|$-torsion free and $\operatorname{tr}(R)$ is nilpotent, then $R$ is nilpotent. A consequence of this theorem is that if $R$ is semi-prime, with no additive $|G|$-torsion, then $R^{G}$ is also semi-prime [10].

Proposition 2.3. If $G$ is a finite group of automorphisms of $R$ such that $R$ is $|G|$-torsion free, then $P\left(R^{G}\right)=P(R) \cap R^{G}$.

Proof. Using $A=R^{G}$ in Lemma 2.2, we have $P(R) \cap R^{G} \subseteq P\left(R^{G}\right)$. Thus we need only show that $P\left(R^{G}\right) \cong P(R)$. Assume this is false. Then in $\bar{R}=R / P(R), P\left(\overline{R^{G}}\right) \neq(0)$. It is straightforward to check that $\overline{P\left(R^{G}\right)} \subseteq \overline{P\left(R^{G}\right)}$.

Since $P(R)$ is $G$-invariant, we have an induced group $\bar{G}$ acting on $\bar{R} . \quad \bar{R}$ is $n$-torsion free by Lemma 2.1 , so by the consequence of Bergman and Isaacs' theorem mentioned above, $\bar{R}^{\bar{G}}$ is semi-prime since $\bar{R}$ is. Let $\bar{N}$ be a nonzero nilpotent ideal of $\overline{R^{G}}$, and let $\bar{M}=$ $\left\{\bar{x} \in \bar{R}^{\bar{G}} \mid n^{k} \bar{x} \in \bar{N}\right.$, for some $\left.k \geqq 0\right\}$. Clearly $\bar{M}$ is nilpotent, and $\bar{M}$ is an ideal of $\bar{R}^{\bar{G}}$ since $n \bar{R}^{\bar{G}} \cong \bar{R}^{\bar{G}}$. Since $\bar{M} \supseteqq \bar{N} \neq(0)$, this contradicts $\bar{R}^{\bar{G}}$ being semi-prime. Thus, $P\left(R^{G}\right) \subseteq P(R)$ and we are done.

Lemma 2.4. Let $R$ be prime, and let $(0) \neq I$ be a G-invariant ideal of $R$. If $a \in R^{G}$ such that $a I^{G} a=0$, then $a=0$.

Proof. As in Corollary 1.3, let $H$ be the subgroup of automorphisms in G. By Proposition 2.3, $R^{H}$ is semi-prime since $P\left(R^{H}\right)=$ $P(R) \cap R^{H}=(0)$. Also, $I^{H}$ is an ideal of $R^{H}$. It will suffice to show that $a I^{H}=(0)$, for then since $a \in R^{H}, \operatorname{tr}_{H}(a I)=a \operatorname{tr}_{H}(I) \subseteq a I^{H}=(0)$. Thus $a I$ is a ring with no fixed elements under $H$, so by Bergman and Isaacs' theorem [2], $a I$ is nilpotent. Since $R$ is prime, $a I=(0)$, and we then have $a=0$.

If $H=G$, then $a I^{G} a=(0)=a I^{H} a$ implies that $a I^{H}$ is a nilpotent right ideal of $R^{H}$, which contradicts $R^{H}$ semi-prime, unless $a I^{H}=(0)$, and we are done by the above. We may therefore assume that 
[G:H] $=2$, and that $R^{H}$ has an involution * with $R^{G}=S_{R^{H}}=S$. Now for any $x \in I^{H}$, we have $x a x^{*} \in S \cap I^{H}=I^{G}$. Thus $a x a x^{*} a=0$, and also $a\left(x+x^{*}\right) a=0$. Then $a x a=-a x^{*} a$, and so $a x a x a=0$. This implies $(a x)^{3}=0$, and thus $a I^{H}$ is a nil right ideal of $R^{H}$ of bounded index. By Levitzki's theorem $[4$, p. 1$], R^{H}$ has a nilpotent ideal, a controdiction unless $a I^{H}=(0)$. We are done.

THEOREM 2.5. Let $R$ be semi-prime, and let $I$ be a G-invariant ideal of $R$ such that for some $a \in R^{G}, a I^{G} a=0$. Then $a I a=(0)$.

Proof. We proceed by induction on $|G|$. If $|G|=1$, there is nothing to prove. So, assume true for all groups $K$ with $|K|<|G|$. Since $R$ is semi-prime and $n$-torsion free, $(0)=\bigcap_{\alpha} P_{\alpha}$, where the $P_{\alpha}$ are prime ideals such that $R / P_{\alpha}$ is $n$-torsion free, for each $\alpha$, by Lemma 2.1. To show that $a I a=(0)$, it will suffice to show that $a I a \subseteq P_{\alpha}$, for all $\alpha$.

First consider the case when $P_{\alpha}=P$ is $G$-invariant. Then $\bar{R}=$ $R / P$ is prime, with induced group $\bar{G}$, and $\bar{a} \bar{I}^{\bar{G}} \bar{a}=(0)$. Since $n \bar{I}^{\bar{G}} \cong \overline{I^{G}}$, we have $\bar{a} \bar{I}^{\bar{\sigma}} \bar{a}=(0)$. Now by Lemma 2.4 , either $\bar{I}=(0)$ or $\bar{a}=(0)$, and so either $I \subseteq P$ or $a \in P$. In any event, $a I a \cong P$.

Now say that $P_{\alpha}=P$ is not $G$-invariant, and let $J=\bigcap_{g \in G} P^{g}$. Each $P^{g}$ is a prime ideal of $R$ by Corollary 1.2 , and so $\bar{R}=R / J$ is a semi-prime ring, with an induced group $\bar{G}$ since $J$ is $G$-invariant. As above, we have that $\bar{a} \bar{I}^{\bar{\sigma}} \bar{a}=(0)$ in $\bar{R}$, and so without loss of generality we may simply assume that $\bigcap_{g \in G} P^{g}=(0)$.

Let orb $P=\left\{P^{g} \mid g \in G\right\}$. Let $m$ be the smallest integer such that, for any choice of $m$ distinct members of orb $P$, say $P_{1}, \cdots, P_{m}$, we have $a\left(I \cap P_{1} \cap P_{2} \cap \cdots \cap P_{m}\right) a=(0)$. Since $P_{1} \cap \cdots \cap P_{n}=(0)$, clearly $m \leqq n$. If $m=1$, then $a(I \cap P) a=0$. Choose $P_{i} \in \operatorname{orb} P, P_{i} \neq P$, and pass to $\bar{R}=R / P_{i}$. Then $\bar{a}(\bar{I} \bar{P}) \bar{a}=(\overline{0})$ in $\bar{R}$, a prime ring. Now if $\bar{a} \neq \overline{0}$, then $\bar{I} \bar{P}=(\overline{0})$. Since $\bar{R}$ is prime and $\bar{P} \neq(\overline{0})$, it must be that $\bar{I}=(\overline{0})$. Thus, either $a \in P_{i}$ or $I \subseteq P_{i}$. But then, since $a \in R^{a}$, if $a \in P_{i}$ then $a \in \bigcap_{g \in G} P_{i}^{g}=(0)$, and if $I \cong P_{i}$ then $I \subseteq \bigcap_{g \in G} P_{i}^{g}=(0)$. That is either $a=0$ or $I=(0)$. In either case, $a I a=(0)$, and we are done.

We may therefore assume that $m>1$. By the minimality of $m$, there exist $m-1$ distinct members $P_{1}, P_{2}, \cdots, P_{m-1}$ in orb $P$ such that if $V=P_{1} \cap \cdots \cap P_{m-1}$, then $a(I \cap V) a \neq(0)$. Let $K=\{g \in G \mid g$ permutes the $\left.m-1 P_{i}^{\prime} s\right\}$. If $K=G$, we have a contradiction since $G$ is transitive on orb $P$, and $m-1<n$. Therefore $K$ is a proper subgroup of $G$. Since $|K|$ divides $|G|, R$ is $|K|$-torsion free and $I \cap V$ is a $K$-invariant ideal of $R$ with $a \in R^{K}$. Now choose $x, y \in I \cap V$.






$$
0=a\left(\sum_{\sigma \in K} x^{\sigma}\right) \alpha+\sum_{\sigma \notin K} a x^{o} \alpha=a \operatorname{tr}_{K}(x) a+\alpha c(x) \alpha,
$$

where $c(x)=\sum_{\sigma \notin K} x^{\sigma}$. If $\sigma \notin K$, then for some $P_{i}, P_{i}^{\sigma} \notin\left\{P_{1}, \cdots, P_{m-1}\right\}$. Thus $x^{\sigma} \in P_{i}^{\sigma}$, and so $y a x^{\sigma} a y \in(I \cap V) \cap P_{i}^{\sigma}$. Letting $P_{i}^{\sigma}=P_{m}$, it follows that $a y \alpha x^{\sigma} \alpha y \alpha \in \alpha\left(I \cap P_{1} \cap \cdots \cap P_{m}\right) \alpha=(0)$. Then $\left(\alpha x^{\sigma} \alpha y\right)^{3}=0$, for all $y \in I \cap V$, and so $a x^{\sigma} a(I \cap V)$ is a nil right ideal of bounded index in $R$. Since $R$ is semi-prime, we must haue $a x^{\sigma} \alpha(I \cap V)=(0)$. Thus, $a c(x) \alpha(I \cap V)=(0)$. It follows that $\alpha \operatorname{tr}_{K}(x) \alpha(I \cap V)=(0)$, also. Now $a \operatorname{tr}_{K}(x) a \in I \cap V$, and since $I \cap V$ is itself a semi-prime ring (being an ideal in $R$ ), we must have $a \operatorname{tr}_{K}(x) a=0$, for all $x \in I \cap V$.

Since $n(I \cap V)^{K} \cong \operatorname{tr}_{K}(I \cap V)$, we have shown that $a(I \cap V)^{K} \alpha=$ (0). We may now apply induction on the order of $K$ to see that $a(I \cap V) a=(0)$. This is a contradiction.

If $A$ is a Jordan ring, we say that an element $a \in A$ is an absolute zero divisor in $A$ if $A U_{a}=(0)$. It is known that if $A$ has no absolute zero divisors, then $A$ is semi-prime [12].

CoRollary 2.6. If $R$ is semi-prime, then $R^{G}$ is semi-prime. Moreover, $R^{G}$ has no absolute zero divisors.

Proof. Using $I=R$ in Theorem 2.5, we see that $R^{G}$ has no absolute zero divisors. By the above, $R^{G}$ is semi-prime.

CoRollary 2.7. If $I$ is a semi-prime ideal of $R$ such that $R / I$ is n-torsion free, then $I \cap R^{G}$ is a semi-prime (Jordan) ideal of $R^{G}$.

Proof. Let $J=\bigcap_{\sigma \in G} I^{o} . \quad J$ is $G$-invariant, and is an ideal of $R$ since each $I^{\sigma}$ is an intersection of prime ideals by Corollary 1.2. Then $\bar{R}=R / J$ is a semi-prime ring with induced group $\bar{G}$, and $\bar{R}$ is $n$ torsion free since $R / I^{\sigma}$ is $n$-torsion free, for all $\sigma$. By Corollary 2.6, $\bar{R}^{\bar{G}}$ is semi-prime. As in the proof of Proposition 2.3, it follows that $\overline{R^{G}}$ is semi-prime. But, $\overline{R^{G}}=R^{G}+J / J \cong R^{G} / R^{G} \cap J=R^{G} / R^{G} \cap I$, where $\cong$ indicates an isomorphism as Jordan ${ }^{+}$rings. Thus $R^{G} \cap I$ is a semiprime ideal of $R^{G}$.

THEOREM 2.8. Let $G$ be a finite group of Jordan automorphisms of a ring $R$, such that $R$ has no additive $|G|$-torsion. Then $P(R) \cap R^{G}=$ $P\left(R^{G}\right)$.

Proof. That $P(R) \cap R^{G} \cong P\left(R^{G}\right)$ is just Lemma 2.2. To see $P\left(R^{G}\right) \subseteq P(R)$, use the fact that $P(R)=\bigcap_{\alpha} I_{\alpha}$, where the $I_{\alpha}$ are prime ideals of $R$ such that $R / I_{\alpha}$ is $n$-torsion free. By Corollary 2.7, $I_{\alpha} \cap R^{G}$ 
is a semi-prime ideal of $R^{G}$, for all such $\alpha$. Thus $P\left(R^{G}\right) \subseteq \bigcap_{\alpha}\left(I_{\alpha} \cap R^{G}\right) \subseteq$ $\left(\bigcap_{\alpha} I_{\alpha}\right) \subset P(R)$.

3. The Jacobson radical. In this section we show that the Jacobson radical of $R^{G}$ is the intersection with $R^{G}$ of the Jacobson radical of $R$. When $G$ consists of an involution, this has already been proved by K. McCrimmon [9], and when $G$ is a finite group of automorphisms of $R$, this has been proved by Montgomery [10]. An additional assumption will be needed concerning $n=|G|$ and the additive group of $R$; that is $n R=R$. Together with our previous assumption that $n x=0$ implies $x=0$, this says that $n$ is a bijection on $R$. Such a hypothesis is needed, since by an example of Martindale [8], the theorem is false for automorphisms if one only assumes that $R$ has no $n$-torsion. Moreover, the hypothesis that $n$ is a bijection is used in the proof of Montgomery's result, which we shall need here.

In a Jordan ring $A$, the Jacobson radical $J(A)$ is defined as the maximal quasi-regular ideal, where an element $x \in A$ is quasi-regular if $1-x$ is invertible (if $1 \notin A$, the inverse is formal). When $A$ is a special Jordan ring, say $A \subseteq R^{+}$, where $R$ is an associative ring, then being invertible in the Jordan sense is the same as being invertible in the associative sense. Thus $x$ is quasi-regular in $A$ if and only if there exists $y \in A$ such that $x+y+x y=0$, and $x y=y x$. We also denote the Jacobson radical of $R$ by $J(R)$; since $J(R)=J\left(R^{+}\right)$ by a theorem of McCrimmon [9], there will be no ambiguity in this notation.

We first note that Jordan automorphisms preserve primitive ideals.

LEMMA 3.1. Let $\phi$ be a Jordan automorphism of $R$, and let $P$ be a (right) primitive ideal of $R$. Then $P^{\phi}$ is a (right or left) primitive ideal of $R$.

Proof. Since $P$ is prime, we may apply Corollary 1.2 to see that $R / P$ and $R / P^{\phi}$ are either isomorphic or anti-isomorphic.

As a consequence of this lemma, when $G$ is a group of Jordan automorphisms and $P$ is a primitive ideal, we seethat $I=\bigcap_{\alpha \in G} P^{\sigma}$ is a semi-simple ideal of $R$.

The first two parts of the next lemma are actually a special case of [6; Lemma 1, p. 3.3], where it is proved that if $I$ is an inner ideal of a Jordan ring, and $w \in I$ is quasi-regular, then $w$ is quasi-regular in $I$. However, as the proof in our case is very elementary, we include it for the sake of completeness.

Lemma 3.2. Let $A$ be a Jordan subring of a ring $R$. 
(1) Let $I$ be a Jordan ideals of $A$. If $w \in I$ is quasi-regular in $A$, it is quasi-regular in $I$.

(2) Let $a \in A$. If $w \in a A a$ is quasi-regular in $A$, it is quasiregualr in $a A a$.

(3) Let $a \in R$. If $a x a \in a R a$ is quasi-regular, then $\alpha^{2} x$ is quasiregular.

Proof. (1) Say that $z \in A$ is the quasi-inverse for $w$. Then $(1-w)(1-z)(1-w)=(1-w)$. This gives $1-w+w^{2}+w z+z w-$ $w-z-w z w=1-w$, and so $z=w^{2}+w z+z w-w-w z w \in I$.

(2) Say that $w=a x a$, for some $x \in A$, and that $z$ is the quasiinverse for $w$. Then $a x a+z+a x a z=0$, and $a x a z=z a x a$. One can check that

$$
z=-a x a-a x a z=a\left(-x+x a^{2} x+x a w a x\right) \alpha \in a A a .
$$

(3) By part (2), the quasi-inverse of $a x a$ is $z=a y a \in a R a$. Thus $a x a+a y a+a x a^{2} y a=0$. Multiplying on the left by $a$, we see that $\left(a^{2} x+a^{2} y+a^{2} x a^{2} y\right) a=0$. Letting $z=a^{2} x+a^{2} y+a^{2} x \alpha^{2} y$, we see that $z^{2}=0$. Thus $z$ is quasi-regular, with quasi-inverse $-z$. Writing $w+z+w z=w \circ z$, we see that

$$
a^{2} x \circ\left(a^{2} y \circ(-z)\right)=\left(a^{2} x \circ a^{2} y\right) \circ(-z)=z \circ(-z)=0 .
$$

Thus $a^{2} x$ is right quasi-regular. Similarly, $a^{2} x$ is left quasi-regular.

Before proceeding, we observe several consequences of our hypothesis that $|G|$ is a bijection. First, if $P$ is any proper prime ideal of $R$, then $|G|$ is a bijection on $\bar{R}=R / P$. For, $n \bar{R}=\bar{R}$ since $n R=R$, and if $\bar{R}$ has any $n$-torsion, then $n \bar{R}=\overline{0}$ since $\bar{R}$ is prime. This says that $n R \subseteq P$, but then $R \subseteq P$, a contradiction. Now if $\bar{R}$ is any semi-prime homomorphic image of $R,|G|$ will be a bijection on $\bar{R}$. If $\bar{R}=R / I$ where $I$ is $G$-invariant, so that $\bar{R}$ has the induced group of automorphisms $\bar{G}$, it follows that $\bar{R}^{\bar{G}}=\bar{R}^{\bar{G}}$ (see the discussion at the end of the introduction).

We are now able to finish the case of radical rings.

Lemma 3.3. Assume that $|G|$ is a bijection on $R$, and that $J\left(R^{G}\right)=R^{G}$. Then $J(R)=R$.

Proof. We proceed by induction on $n=|G|$. If $n=1$, then $R=R^{G}$ and there is nothing to prove. Thus, assume that for any group $K$ of Jordan automorphisms with $|K|<|G|$ and $R^{K}$ a radical ring, it follows that $J(R)=R$.

To show that $J(R)=R$, we show that $R$ has no proper primitive 
ideals. Say that $P$ is such an ideal. If $P$ is $G$-invariant, then $\bar{R}=R / P$ has induced group $\bar{G}$, and $\bar{R}^{\bar{G}}=\bar{R}^{\bar{G}}$ by the remarks above. Thus $\bar{R}^{\bar{G}}=J\left(\bar{R}^{\bar{G}}\right)$. We may therefore reduce to the case when $R$ is primitive. Apply Corollary 1.3, with $H$ the subgroup of automorphisms. Now $J\left(R^{H}\right)=J(R) \cap R^{H}$ by Montgomery's theorem [10], and $J\left(R^{G}\right)=J\left(S_{R^{H}}\right)=J\left(R^{H}\right) \cap R^{G}$ by McCrimmon's theorem [9]. Since $J(R)=(0)$, it follows that $R^{G}=J\left(R^{G}\right)=(0)$. This contradicts the theorem of Bergman and Isaacs [2], since $R$ is primitive and so cannot be nilpotent.

We may therefore assume that $P$ is not $G$-invariant. By Lemma 3.1, $I=\bigcap_{g \in G} P^{g}$ is a semi-simple ideal of $R$. As in the previous case, we may pass to $\bar{R}=R / I$, and so assume that $R$ is semi-simple with $\bigcap_{g \in G} P^{g}=(0)$.

Let orb $P=\left\{P^{g} \mid g \in G\right\}$, and let $m$ be the smallest positive integer such that, for any choice of $m$ distinct members of orb $P$, say $P_{1}, P_{2}$, $\cdots, P_{m}$, we have $\bigcap_{i=1}^{m} P_{i}=(0)$. Clearly $m \leqq n$. If $m=1$, then $P=$ (0). This says that $P$ is $G$-invariant, a contradiction. We may therefore assume that $m>1$. We proceed as in the proof of Theorem 2.5: by the minimality of $m$, there exist $m-1$ distinct members $P_{1}, P_{2}, \cdots, P_{m-1}$ of orb $P$ such that $V=\bigcap_{i=1}^{m-1} P_{i} \neq(0)$, and we let $K$ be the set of $g \in G$ which permute $\left\{P_{1}, \cdots, P_{m-1}\right\}$. If $K=G$, we have a contradiction since $G$ is transitive on orb $P$ and $m-1<n$. Thus, $K$ is a proper subgroup of $G$. Since $|K|$ divides $|G|,|K|$ is a bijection on $R$. In fact, $|K|$ is a bijection on $V$. For, clearly $V$ has no $|K|-$ torsion, and since $R / V$ is semi-prime, $|K|$ is a bijection on $R / V$. In particular, $R / V$ is $|K|$-torsion free, from which it follows that $|K| V=$ $V$. Now $V$ is a $K$-invariant ideal of $R$, so if we can show that $J\left(V^{K}\right)=V^{K}$, it will follow by induction on $|K|$, using the ring $V$, that $J(V)=V$. Since $R$ is a semi-simple ring, it can have no quasiregular ideals, and thus $V=(0)$, a contradiction. Thus, the theorem will be proved if we can show that $J\left(V^{K}\right)=V^{K}$.

Choose $x \in V$. Then $\operatorname{tr}(x)=\operatorname{tr}_{K}(x)+c(x)$, where $c(x)=\sum_{g \notin K} x^{g}$. One can check that $\operatorname{tr}(V)=\{\operatorname{tr}(x) \mid x \in V\}$ is a Jordan ideal of $R^{G}$, and thus by Lemma 3.2, (1), the quasi-inverse of $\operatorname{tr}(x)$ also lies in $\operatorname{tr}(V)$. Say that $\operatorname{tr}(y)$, for $y \in V$, is the quasi-inverse of $\operatorname{tr}(x)$. Now $\operatorname{tr}(y)=\operatorname{tr}_{K}(y)+c(y)$, and so

$$
\begin{aligned}
0= & \operatorname{tr}(x)+\operatorname{tr}(y)+\operatorname{tr}(x) \operatorname{tr}(y) \\
= & \operatorname{tr}_{K}(x)+\operatorname{tr}_{K}(y)+\operatorname{tr}_{K}(x) \operatorname{tr}_{K}(y)+c(x)+c(y)+c(x) c(y) \\
& +\operatorname{tr}_{K}(x) c(y)+c(x) \operatorname{tr}_{K}(y) .
\end{aligned}
$$

If $g \notin K$, then for some $P_{i}, P_{i}^{g} \notin\left\{P_{1}, \cdots, P_{m-1}\right\}$. Thus $x^{g} \in P_{i}^{g}$ and $x^{g} V=$ $V x^{g}=(0)$ since $x^{g} V \cong P_{i}^{g} \cap\left(P_{1} \cap \cdots \cap P_{m-1}\right)=(0)$ by the mimimality of $m$. Similarly $y^{g} V=V y^{g}=(0)$. Multiplying the above expression 
on the right by any $w \in V$, we get $(0)=0 \cdot V=\left[\operatorname{tr}_{K}(x)+\operatorname{tr}_{K}(y)+\right.$ $\left.\operatorname{tr}_{K}(x) \operatorname{tr}_{K}(y)\right] V$. Since $V$ is a semi-simple ring, it has no absolute zero-divisors, and thus $\operatorname{tr}_{K}(x) \circ \operatorname{tr}_{K}(y)=0$. Thus, every element of $V^{K}$ is quasi-regular, so $J\left(V^{K}\right)=V^{K}$, which is what was needed.

LEMma 3.4. Let I be a (Jordan) ideal of the special Jordan ring $A$ such that $a^{2}=0$, for all $a \in I$. If $A$ has no absolute zero-divisors, then $I=(0)$.

Proof. If $x \in A$, and $a \in I$, then $a x+x a \in I$, and so $(a x+x a)^{2}=$ 0 . This gives $a x a x+a x^{2} a+x a x a=0$ since $a^{2}=0$. Multiplying by $a, a x a x a=(0)$. Linearizing on $x$ and then multiplying on the right by $x a$, we have axayaxa $=(0)$, for all $x, y \in A$. Since $A$ has no absolute zero-divisors, we see that $a x a=0$, all $x \in A$, and thus $a=0$. Thus, $I=(0)$.

THEOREM 3.5. Assume that $|G|$ is a bijection on $R$, and that $R$ is semi-simple. Then $R^{G}$ is semi-simple.

Proof. Choose any $a \in J\left(R^{a}\right)$ and consider the ring $a R a$, which is $G$-invariant. $(a R a)^{G}=a R^{G} a \subseteq J\left(R^{G}\right)$, so every element of $a R^{G} a$ is quasi-regular. By Lemma 3.2, (2), $a R^{G} a=J\left(a R^{G} a\right)$ so we may apply Lemma 3.3 to the ring $a R a$ to see that $J(a R a)=a R a$. But now, by Lemma 3.2, part (3), $a^{2} R$ is a quasi-regular right ideal of $R$, a contradiction unless $a^{2}=(0)$. Thus $a^{2}=(0)$ for all $a \in J\left(R^{a}\right)$.

But $R^{G}$ has no absolute zero-divisors, by Corollary 2.6. Applying Lemma 3.4 with $A=R^{G}$, we see that $J\left(R^{G}\right)=(0)$.

Corollary 3.6. When $|G|$ is a bijection on $R, J\left(R^{G}\right)=J(R) \cap R^{G}$.

Proof. Using Theorem 2.5, the proof now follows exactly as in the associative situation [10].

4. Polynomial identities. In this section we prove that if $R^{G}$ satisfies a polynomial identity $(P I)$, then $R$ also satisfies a $P I$. When $G$ consists of an involution, this has been proved by Amitsur [1], and when $G$ consists of automorphisms, this has been proved by V. K. Kharchenko [7] under the assumption that $R$ has no $|G|$-torsion. When $R$ is semi-prime, both Amitsur and Kharchenko obtained a bound on the degree of the identity satisfied by $R$ : namely, if $n=$ $|G|$ and $R^{G}$ satisfies and identity of degree $d$, then $R$ satisfies the standard identity $S_{n d}[x]$ of degree $n d$. We will show that the same conclusion holds when $G$ is a finite group of Jordan automorphisms. We assume throughout that $R$ has no $|G|$-torsion. This assumption 
is necessary, for Bergman and Isaacs have produced an example when $G$ is a finite group of automorphisms, $R^{G}=(0)$ but $R$ satisfies no identity at all, when $|G| R=(0)$ [2]. We will also assume, for the sake of simplicity, that all identities have integer coefficients, and at least one monomial of highest degree has coefficient \pm 1 . With care, more general coefficients may be allowed.

LEMMA 4.1. Let $R$ be prime, and say that $R^{G}$ satisfies a $P I$ of degree $d$. Then $R$ satisfies $S_{n d}[x]$.

Proof. As in previous sections, we use Corollary 1.3 to see that $R^{G}=S_{R^{H}}$, where $R^{H}$ is the subring of $R$ fixed by the subgroup $H$ of automorphisms of $G$. Since $R^{H}$ is semi-prime, it will satisfy $S_{2 d}[x]$ by Amitsur's theorem (simply $S_{d}[x]$ if $H=G$ ) and thus $R$ will satisfy $S_{n d}[x]$ by using Kharchenko's theorem.

THEOREM 4.2. Let $R$ be semi-prime, with no $|G|$-torsion. If $R^{G}$ satisfies a PI of degree $d$, then $R$ satisfies the standard identity $S_{n d}[x]$ of degree $n d$, where $n=|G|$.

Proof. Without loss of generality, we may assume that the identity satisfied by $R^{G}$ is multilinear [5, p. 225]. We proceed by induction on $n$. When $n=1, R=R^{G}$ and we are done. So assume the result is true for all groups $K$ with $|K|<|G|$. By Lemma 2.1, $(0)=P(R)=\bigcap_{\alpha} P_{\alpha}$, where the $P_{\alpha}$ are prime ideals of $R$ such that $R / P_{\alpha}$ is $n$-torsion free. Since $R$ is a subdirect product of the $R / P_{\alpha}$, it suffices to show that each $R / P_{\alpha}$ satisfies $S_{n d}[x]$. If $P=P_{\alpha}$ is $G$ invariant, we may as usual pass to $\bar{R}=R / P$, and the result then follows from Lemma 4.1 and the fact that $n \bar{R}^{\bar{G}} \cong \overline{R^{G}}$.

We therefore may assume that $P_{\alpha}$ is not $G$-invariant, and by considering $\bar{R}=R / \cap P_{\alpha}^{g}$, we may reduce to the case where $\bigcap_{g \in G} P^{g}=(0)$. As before, let orb $P=\left\{P^{g} \mid g \in G\right\}$ and let $m$ be the smallest positive integer such that the intersection of any $m$ distinct members of orb $P$ is 0 . If $m=1$, then $P=(0)$, which says that $P$ is $G$-invariant, a contradiction. Thus $1<m \leqq n$.

By the minimality of $m$, there exist $m-1$ distinct members of orb $P$, say $P_{1}, P_{2}, \cdots, P_{m-1}$, such that $V=P_{1} \cap \cdots \cup P_{m-1} \neq(0)$. Let $K=\left\{g \in G \mid g\right.$ permutes the $\left.m-1 P_{i}^{\prime} s\right\}$. As before, $K$ is a proper subgroup of $G$. Regarding $V$ as a semi-prime ring, $K$ acts on $V$ and $V$ has no additive $|K|$-torsion.

Now choose $a_{1}, \cdots, a_{d}, y \in V$. As before, we write $\operatorname{tr}(x)=\operatorname{tr}_{G}(x)=$ $\operatorname{tr}_{K}(x)+c(x)$, where $c(x)=\sum_{g \notin K} x^{g}$, for any $x$. Write the identity as $f\left(x_{1}, \cdots, x_{d}\right)=\sum_{\pi \in S_{d}} \lambda_{\pi} x_{\pi(1)} x_{\pi(2)} \cdots x_{\pi(d)}$. Substitute $x_{i}=\operatorname{tr}\left(a_{i}\right) \in R^{G}$, and premultiply by $y$ : 


$$
\begin{aligned}
0 & =y f\left(\operatorname{tr}\left(a_{1}\right), \cdots, \operatorname{tr}\left(a_{d}\right)\right) \\
& =y \sum_{\pi \in S_{d}} \lambda_{\pi}\left(\operatorname{tr}_{K}\left(a_{\pi(1)}\right)+c\left(a_{\pi(1)}\right)\right) \cdots\left(\operatorname{tr}_{K}\left(a_{\pi(d)}\right)+c\left(a_{\pi(d)}\right)\right) \\
& =y \sum_{\pi} \lambda_{\pi} \operatorname{tr}_{K}\left(a_{\pi(1)}\right) \operatorname{tr}_{K}\left(a_{\pi(2)}\right) \cdots \operatorname{tr}_{K}\left(a_{\pi(d)}\right)+\sum_{l} y u_{l},
\end{aligned}
$$

where each $u_{l}$ is a product of terms, at least one of which is of the form $a^{\sigma}$, where $a \in V$ and $\sigma \notin K$. Since $\sigma \notin K$, there exists $P_{i}$ such that $P_{i}^{\sigma} \notin\left\{P_{1}, \cdots, P_{m-1}\right\}$. Now $a^{\sigma} \in P_{i}^{\sigma}$, and so $y u_{l} \in\left(P_{1} \cap \cdots \cap P_{m-1}\right) \cap$ $P_{i}^{\sigma}=(0)$ by the choice of $m$. Thus $V \cdot f\left(\operatorname{tr}_{K}\left(a_{1}\right), \cdots, \operatorname{tr}_{K}\left(a_{d}\right)\right)=(0)$. Since $V$ has no absolute zero-divisors, and $n V^{K} \subseteq \operatorname{tr}_{K}(V)$, it follows that $f\left(b_{1}, \cdots, b_{d}\right)=0$ for all choices of $b_{i} \in V^{K}$. That is, $V^{K}$ satisfies a $P I$ of degree $d$. We may now apply induction to $K$ acting on $V$, and conclude that $V$ satisfies the standard identity of degree $d|K|<$ $d|G|$.

To complete the proof, we note that since $V \neq(0), V \nsubseteq P^{g}$, for some $g \in G$. The prime ring $R / P^{g}$ thus contains a nonzero ideal $V+P^{g} / P^{g}$ which satisfies the standard identity of degree $d n$. Since an ideal in a prime ring has the same ring of quotients as the prime ring, when the ideal satisfies a $P I$, it follows that $R / P^{g}$ also satisfies $S_{d n}[x]$. Now for any other $h \in G, R / P^{h}$ is either isomorphic or antiisomorphic to $R / P^{g}$ by Corollary 1.2 , and thus $R / P^{h}$ also satisfies $S_{d_{n}}[x]$. In particular, $R / P$ satisfies $S_{d n}[x]$, and we are done.

COROLlary 4.3. Assume that $R^{G}$ satisfies a PI of degree $d$, and that $R$ has no $|G|$-torsion. Then for some positive integer $k, R$ satisfies $\left(S_{|G| d}[x]\right)^{k}$.

Proof. Follow exactly the argument given by Amitsur for the case of an involution [1, proof of Theorem 6], using the complete direct product of copies of $R$ and Theorem 4.2 above.

5. Nil ideals of bounded index. We have two major objectives in this section: to show that if $R^{G}$ is nil of bounded index, then so is $R$, and to show that if $R^{g}$ is semi-prime, then it has no nonzero (Jordan) ideals which are nil of bounded index. The proof of the first statement follows the same general outline as our previous arguments.

Lemma 5.1. If $R$ is prime, then $R^{G}$ cannot be nil of bounded index.

Proof. As in Corollary 1.3, let $H$ be the subgroup of automorphisms of $G$ and consider $R^{H}$. By Proposition 2.3, $R^{H}$ is semi-prime, so cannot have any ideals which are nil of bounded index. Thus, 
we are done if $H=G$. When $H \neq G$, we have $R^{G}=S_{R^{H}}$. But then, if $R^{G}$ is nil of bounded index $m$, choose $a \in R^{G}$ with $a^{2}=(0)$. For any $x \in R^{H}, x a+a x^{*} \in R^{G}$ so $\left(x a+a x^{*}\right)^{m}=0$. Using $a^{2}=(0)$ and premultiplying by $a$, we see $a(x \alpha)^{m}=0$. Thus $R^{H} a$ is a nil left ideal of bounded index of $R^{H}$, a contradiction unless $a=0$. But then $R^{G}=(0)$, which also contradicts $R^{H}$ being semi-prime.

THEOREM 5.2. If $R^{G}$ is nil of bounded index, and $R$ has no $|G|$-torsion, then $R$ is nil of bounded index.

Proof. We will first show that $R=P(R)$. To show this, we will show that $R$ has no prime ideals. As before, by Lemma 2.1, it will suffice to look at prime ideals $P$ such that $R / P$ is $n$-torsion free. If $P$ is $G$-invariant, we may pass to $\bar{R}=R / P$ and apply Lemma 5.1 to get a contradiction. We may therefore assume that $P$ is not $G$-invariant. The rest of the argument follows closely that of Theorem 4.2: assume $\bigcap_{g \in G} P^{g}=(0)$, let $V=P_{1} \cap \cdots \cap P_{m-1}$, where $m$ is minimal such that the intersection of $m$ members of orb $P$ is zero, define $K$ as before, show that $V^{K}$ is nil of bounded index, and apply the induction hypothesis on $|K|$ to conclnde that $V$ is nil of bounded index. This contradicts the assumption that $R$ is semiprime. Thus $R$ has no prime ideals, so $R=P(R)$.

We now use a variation of Amitsur's argument. Let $R_{1}=$ $\prod_{\alpha \in I} R_{\alpha}$, where each $R_{\alpha}=R$ and the index set $I$ is just $R$ itself. Extend $G$ to $G_{1}$, acting on $R_{1}$ componentwise. Then $R_{1}^{G_{1}}$ is nil of bounded index, so by the previous argument we have $P\left(R_{1}\right)=R_{1}$. In particular, $R_{1}$ is nil. Consider the element $f \in R_{1}$ given by $f(\alpha)=$ $\alpha \in R$. Then for some $k, f^{k}=0$. But this says that $\alpha^{k}=0$, all $\alpha \in R$. That is $R$ is nil of bounded index.

When $G$ is generated by an involution, M. Rich [11] has obtained an explicit bound on the nil index of $R$. He has proved that if $R$ is an algebra over a field $F$, where $F$ has at least $d^{2}$ elements, and $S_{R}$ is nil of index $d$, then $R$ is nil of index $\leqq 2 d$. Hopefully a similar bound can be obtained for any finite group of Jordan autmorphisms.

With a stronger assumption on the characteristic of $R$, more can be said:

COROLlARY 5.3. Let $R$ be an algebra over a fild of characteristic 0 , and assume that $R^{G}$ is nil of bounded index. Then $R$ is nilpotent.

Proof. By Theorem 5.2, $R$ is nil of bounded index. The conclusion now follows by applying the Nagata-Higman theorem [5, p. 274]. 
When $G$ consists of automorphisms, Bergman and Isaacs' theorem (mentioned earlier) asserts that if $R^{G}$ is nilpotent, then $R$ is nilpotent, provided $R$ is $n$-torsion free. We raise the analogous question for groups of Jordan automorphisms:

Question 5.4. Let $G$ be a finite group of Jordan automorphisms of a ring $R$, such that $R$ has no additive $|G|$-torsion. If $R^{G}$ is (Jordan) nilpotent, must $R$ be (Jordan) nilpotent?

A Jordan ring $A$ is nilpotent if for some positive integer $m$, and any $x_{1}, \cdots, x_{m} \in A, x_{1} U_{x_{2}} U_{x_{3}} \cdots U_{x_{m}}=0$. Rich [11] has shown that if a ring $R$ is Jordan nilpotent then it is actually nilpotent in the associative sense; thus, if Question 5.4 can be answered affirmatively, $R$ must actually be nilpotent. The answer to this question is not known even when $G$ consists of an involution *.

We remark, however, that the answer is false when $R$ has $|G|-$ torsion. When $G$ consists of automorphisms, one may use the example of Bergman and Isaacs mentioned at the beginning of $\S 4$.

We now proceed to ideals of $R^{G}$.

THEOREM 5.5. Assume that $R$ is $|G|$-torsion free and that $R^{G}$ is semiprime. Then $R^{G}$ has no nonzero (Jordan) ideals which are nil of bounded index.

Proof. By Theorem 2.8, $P(R) \cap R^{G}=P\left(R^{G}\right)=(0)$, and thus in $\bar{R}=R / P(R), \overline{R^{G}}=R^{G}+P(R) / P(R) \cong R^{G}$. Since $n \bar{R}^{\bar{G}} \cong \overline{R^{G}}$, it follows that $\bar{R}^{\bar{G}}$ is also semi-prime. Let $I$ be a nil ideal of $R^{G}$ of bounded index, and let $\bar{I}$ be the image of $I$ in $\bar{R}$. Let $\bar{I}_{1}=\left\{\bar{x} \in \bar{R}^{\bar{G}} \mid n^{k} \bar{x} \in \bar{I}\right.$, for some integer $k \geqq 0\}$. Then $\bar{I}_{1}$ is a Jordan ideal of $\bar{R}^{\bar{G}}$ which is nil of bounded index, since $\bar{R}$ is $n$-torsion free. Since $\bar{I}_{1} \supseteq \bar{I} \cong I, \bar{I}_{1} \neq$ (0). Thus, without loss of generality, we may assume that $R$ is semi-prime.

Choose any $a \in I$, and consider the ring $a R a$. Since $a \in R^{G}, a R a$ is $G$-invariant, and $(\alpha R a)^{G}=\alpha R^{G} a \subseteq I$. Thus $\alpha R^{G} a$ is nil of bounded index. By Theorem 5.2, $a R a$ is nil of bounded index. But then $a^{2} R$ is a nil right ideal of bounded index in $R$. Since $R$ is semi-prime, this is impossible by Levitzki's theorem, unless $a^{2}=0$. Thus $\alpha^{2}=0$, for all $a \in I$. By Lemma 3.4, $I=(0)$, and we are done.

\section{REFERENCES}

1. S. A. Amitsur, Rings with involution, Israel J. Math., 6 (1968), 99-106.

2. G. M. Bergman and I. M. Isaacs, Rings with fixed-point-free group actions, Proc. 
London Math. Soc., 27 (1973), 69-87.

3. T. S. Frickson and S. Montgomery, The prime radical in special Jordan rings, Trans. Amer. Math. Soc., 156 (1971), 155-164.

4. I. N. Herstein, Topics in Ring Theory, University of Chicago Press, 1969.

5. N. Jacobson, Structure of Rings, Amer. Math. Soc., Colloquium Publ. 37, revised edition 1964.

6. N. Jacobson, Lectures on Quadratic Jordan Algebras, Tata Institute, Bombay, 1969.

7. V. K. Kharchenko, Galois extensions and quotient rings, Algebra i Logika, 13 (1974), 460-484.

8. W. S. Martindale, III, Fixed rings of automorphisms and the Jacobson radical,

J. London Math. Soc., (to appear).

9. Kevin McCrimmon, On Herstein's theorems relating Jordan and associative algebras, J. Algebra, 13 (1969), 382-392.

10. Susan Montgomery, The Jacobson radical and fixed rings of automorphisms, Communications in Algebra, 4 (1976), 459-465.

11. Michael Rich, The Levitzki radical in associative and Jordan rings, J. of Algebra, 40 (1976), 97-104.

12. C. Tsai, The prime radical in a Jordan ring, Proc. Amer. Math. Soc., 19 (1968), $1171-1175$.

Received November 4, 1976 and in revised form March 16, 1977. The work of the first author was supported by NSF Grant MCS 76-08955 and the work of the second author was supported by NSF Grant MCS 76-07240.

UNIVERSITY OF MASSACHUSETTS

AMHERST, MA 01002

AND

University of Southern California

Los Angeles, CA 90007 


\title{
PACIFIC JOURNAL OF MATHEMATICS
}

\author{
EDITORS
}

RICHARD ARENS (Managing Editor)

University of California

Los Angeles, California 90024

C. W. CurTis

University of Oregon

Eugene, OR 97403

C. C. MOORE

University of California

Berkeley, CA 94720
J. DUgundJI

Department of Mathematics

University of Southern Californı

Los Angeles, California 90007

R. Finn and J. Milgram

Stanford University

Stanford, California 94305

\section{ASSOCIATE EDITORS}

E. F. BECKENBACH

\section{SUPPORTING INSTITUTIONS}

\author{
UNIVERSITY OF BRITISH COLUMBIA \\ CALIFORNIA INSTITUTE OF TECHNOLOGY \\ UNIVERSITY OF CALIFORNIA \\ MONTANA STATE UNIVERSITY \\ UNIVERSITY OF NEVADA, RENO \\ NEW MEXICO STATE UNIVERSITY \\ OREGON STATE UNIVERSITY \\ UNIVERSITY OF OREGON \\ OSAKA UNIVERSITY
}

UNIVERSITY OF SOUTHERN CALIFORNIA

STANFORD UNIVERSITY

UNIVERSITY OF TOKYO

UNIVERSITY OF UTAH

WASHINGTON STATE UNIVERSITY

UNIVERSITY OF WASHINGTON
AMERICAN MATHEMATICAL SOCIETY
NAVAL WEAPONS CENTER 


\section{Pacific Journal of Mathematics \\ Vol. 72 , No. 1 \\ January, 1977}

Kazuo Anzai and Shiro Ishikawa, On common fixed points for several

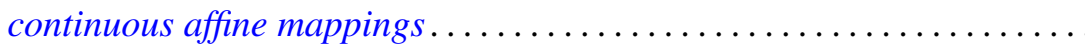

Bruce Alan Barnes, When is a representation of a Banach $*$-algebra

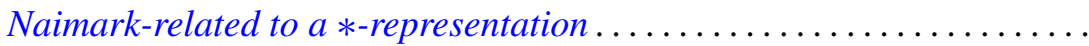

Richard Dowell Byrd, Justin Thomas Lloyd, Franklin D. Pedersen and

James Wilson Stepp, Automorphisms of the semigroup of finite

complexes of a periodic locally cyclic group ...................

Donald S. Coram and Paul Frazier Duvall, Jr., Approximate fibrations and a

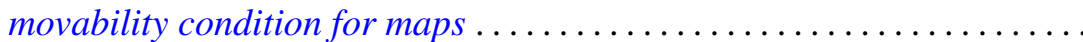

Kenneth R. Davidson and Che-Kao Fong, An operator algebra which is not

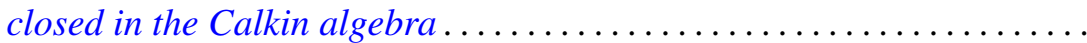

Garret J. Etgen and James Pawlowski, A comparison theorem and oscillation criteria for second order differential systems .............

Philip Palmer Green, $C^{*}$-algebras of transformation groups with smooth

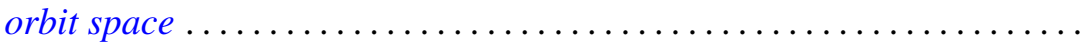

Charles Allen Jones and Charles Dwight Lahr, Weak and norm approximate



G. K. Kalisch, On integral representations of piecewise holomorphic

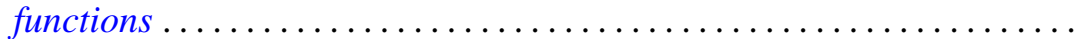

Y. Kodama, On product of shape and a question of Sher

Heinz K. Langer and B. Textorius, On generalized resolvents and

$Q$-functions of symmetric linear relations (subspaces) in Hilbert

space ...................................

Albert Edward Livingston, On the integral means of univalent, meromorphic functions

Wallace Smith Martindale, III and Susan Montgomery, Fixed elements of

Jordan automorphisms of associative rings ..........

R. Kent Nagle, Monotonicity and alternative methods for nonlinear boundary value problems ........................

Richard John O'Malley, Approximately differentiable functions: the $r$ topology.

Mangesh Bhalchandra Rege and Kalathoor Varadarajan, Chain conditions

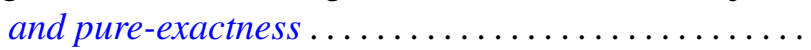

Christine Ann Shannon, The second dual of $C(X)$. .

Sin-ei Takahasi, A characterization for compact central double centralizers of $C^{*}$-algebras .

Theresa Phillips Vaughan, A note on the Jacobi-Perron algorithm. . .

Arthur Anthony Yanushka, A characterization of $\operatorname{PSp}(2 m, q)$ and

$\mathrm{P} \Omega(2 m+1, q)$ as rank 3 permutation groups ......... 